\title{
Caenorhabditis elegans as a model organism for RYR1 variants and muscle ageing
}

\author{
Kathie Nicoll Baines,' Marie-Anne Shaw, lan A Hope \\ From 33rd Annual Meeting of the European Malignant Hyperthermia Group (EMHG) \\ Würzburg, Germany. 15-17 May 2014
}

\begin{abstract}
Background
Malignant hyperthermia $(\mathrm{MH})$, central core disease (CCD), exertional heat stroke (EHS) and late-onset axial myopathy have been attributed to mutations in ryanodine receptor type 1 ( $R Y R 1)$. The RyR1 protein is over 5000 amino acid residues long, making manipulation of the mammalian gene difficult. The ryanodine receptor in Caenorhabditis elegans is UNC-68, which has $40 \%$ amino acid identity to the human protein.
\end{abstract}

\section{Material and methods and results}

Due to the compact nature of the C. elegans genome, the $u n c-68$ gene is only $27 \mathrm{~kb}$ and is entirely contained in the fosmid clone WRM069cA02. Using recombineering, single base pairs were changed in this fosmid to establish nine different variants:

- Four implicated in $\mathrm{MH}$ :

$\circ$ p.G341R c.1021G $>$ A

$\circ$ p.R2163C c.6388G $>$ A

$\circ$ p.R2454H c.7361G>A

$\circ$ pR2458H c.7373G $>$ A

- One implicated in EHS:

$\circ$ p.R163C c.487C > T

- Two implicated in CCD:

$\circ$ p.R4861H c.14582G $>$ A

$\circ$ p.A4940T c.14820G $>$ A

- Two implicated in Late-onset axial myopathy:

$\circ$ p.K3452Q c.10354A $>$ C

$\circ$ pV4849I c.14545G>A

Using these altered fosmids, transgenic strains were developed by microinjection.

In order to establish these strains as a suitable model for studying RYR1 variants phenotyping assays were completed

Malignant Hyperthermia Investigation Unit, St James's University Hospital, LS9 7TF Leeds, UK
Cite this article as: Baines et al: Caenorhabditis elegans as a model organism for RYR1 variants and muscle ageing. BMC Anesthesiology 2014 